\title{
Integridad y conductas inapropiadas en investigación biomédica
}

\section{Integrity and misconduct in biomedical research}

\author{
Luisa Schonhaut B. ${ }^{\mathrm{a}, \mathrm{b}}$
}

aClínica Alemana, Facultad de Medicina, Universidad del Desarrollo, Santiago, Chile

${ }^{b}$ Comité Editorial Revista Chilena de Pediatría

Recibido: 2 de enero de 2019; Aprobado: 17 de enero de 2019

\begin{abstract}
Resumen
Los Comités Editoriales de revistas de corriente principal se ven enfrentados ocasionalmente a conductas éticas inapropiadas en los manuscritos recibidos. El Comité de Ética en las publicaciones (COPE) ofrece recomendaciones para los editores respecto a cómo actuar frente a la sospecha de falta de ética en los manuscritos, ya sea recibidos o publicados. Cuando se pesquisa una mala práctica durante el proceso de revisión por pares, el manuscrito es rechazado, no obstante, si la conducta inapropiada es detectada después de la publicación de manuscrito, se procede a retractar la publicación. Revista Chilena de Pediatría no ha sido exenta a este tipo de conflictos. En este artículo analizamos los distintos aspectos relacionados con la falta de integridad de las publicaciones, como son las autorías, el plagio y el conflicto de intereses. Podemos concluir que las malas prácticas ocurren principalmente por desconocimiento de los autores, más que por intención de fraude. Se espera que el presente manuscrito logre instruir y sensibilizar a nuestros investigadores, respecto a las buenas prácticas en la investigación y publicación, y, contribuir, en lo posible, a prevenir que estas acciones ocurran en los manuscritos enviados a nuestra Revista.
\end{abstract}

Palabras clave: Ética en publicaciones; malas prácticas científicas; plagio; conflicto de intereses; autorías

\section{Keywords:}

Publishing ethics; scientific misconduct; plagiarism; interest conflict; authorship 


\section{Introducción}

El siglo XXI ha traído consigo la explosión del conocimiento y de la información. La redacción y publicación de los manuscritos es el eslabón final del proceso de investigación.

La divulgación en revistas científicas permite expandir el conocimiento, el intercambio de resultados, la generación de redes y la colaboración a nivel nacional e internacional. Además, es un requisito para la carrera académica, postulación a fondos de investigación, otorgando reconocimiento a los investigadores y prestigio a sus instituciones ${ }^{1,2}$. Por este motivo, los investigadores están expuestos a una gran presión por publicar.

Lamentablemente, esta presión aumenta el riesgo de prácticas inapropiadas (Tabla 1). Martinson $\mathrm{BC}^{3}$ reportó que cerca de un tercio de los investigadores que reciben fondos del National Institute of Health (NIH) admitió haber realizado alguna conducta no ética como autorías honorarias, publicaciones duplicadas o redundantes. En un meta-análisis que incluyó 18 estudios de distintas disciplinas, se encontró que 2\% de los investigadores admiten haber falsificado, modificado o inventado datos, cifra que aumentó al $14 \%$ al preguntar por dicha conducta en colegas, siendo más frecuentes entre los médicos y farmacólogos que en otras profesiones.

Con cierta frecuencia los comités editoriales de revistas de corriente principal se ven enfrentados a este tipo de conflictos en los manuscritos recibidos ${ }^{5,6}$, debiendo rechazar el manuscrito cuando se pesquisa una conducta ética inadecuada durante el proceso de revisión por pares, o bien retractar la publicación si la mala práctica es detectada después de la publicación de manuscrito.
El número de manuscritos retractados de Medline ha aumentado considerablemente en las últimas 4 décadas y actualmente representan el $0,02 \%$ de la publicaciones ${ }^{7}$; este aumento se podría deber al incremento de conductas inapropiadas, pero debemos considerar además que la era de la digitalización y la mayor accesibilidad a programas de detección de plagio han su contribuido a su pesquisa, $y$, por otro lado se han facilitado los pasos necesarios para hacer la retracción ${ }^{8}$. Stretton y cols. reportaron que de los manuscritos retractados, $42 \%$ se debieron a plagio, $52 \%$ a falsificación/fabricación y $2 \%$ por disputas de los autores, siendo estas prácticas más prevalentes en países de menores ingre$\operatorname{sos}^{9}$.

El Comité de ética en las publicaciones (COPE por sus siglas en inglés) ${ }^{10}$ fue formado con la misión de regular la ética de las publicaciones en revistas biomédicas, para asegurar que las investigaciones sean conducidas con honestidad, transparencia, confidencialidad y responsabilidad ${ }^{11}$; el COPE ofrece una serie de guías o cartillas de flujo con recomendaciones para los editores respecto a cómo actuar frente a la sospecha de falta de ética en los manuscritos, ya sea recibidos o publicados.

El objetivo del presente manuscrito es revisar los distintos aspectos relacionados con la falta de integridad de las publicaciones, como son las autorías, el plagio y el conflicto de intereses.

\section{Las autorías}

La autoría provee al investigador un crédito individual, que debe ser acorde a su participación en la investigación. Las autorías tienen impacto social, financiero y en las carreras académicas.

Según el Committee of Medical Journal Editors

Tabla 1. Definición de prácticas inapropiadas en orden decreciente de gravedad

\begin{tabular}{ll}
\hline Sub grupo de práctica & Definición \\
\hline Fabricación & Invención de la totalidad o parte de los datos \\
Falsificación & $\begin{array}{l}\text { Distorsión, manipulación de datos } \\
\text { Copia de ideas o palabras sin la adecuada cita ni autorización }\end{array}$ \\
$\begin{array}{l}\text { Flagio } \\
\text { Étta de aprobación del Comité de }\end{array}$ & $\begin{array}{l}\text { Toda investigación original realizada con seres humanos o animales requiere de la } \\
\text { aprobación previa del Comité de Ética }\end{array}$ \\
$\begin{array}{l}\text { Falta de declaración de conflictos de } \\
\text { intereses }\end{array}$ & $\begin{array}{l}\text { No declarar un conflicto de intereses (financiero o no financiero), que puede influir en } \\
\text { juicio profesional y sesgar las conclusiones } \\
\text { Autorías ficticias o fantasmas }\end{array}$ \\
$\begin{array}{l}\text { Los autores invitados son aquellos que no han participado de la investigación y se } \\
\text { agregan a la lista de autores } \\
\text { Los autores fantasmas son investigadores eliminados de la lista de autores para ocultar } \\
\text { un conflicto de interés }\end{array}$ \\
$\begin{array}{ll}\text { Publicación redundante o fraccionada, } \\
\text { "en rebanadas de salame" }\end{array}$ \\
\hline
\end{tabular}


(ICMJE) los criterios de autoría se aplican al investigador que haya contribuido substancialmente en todos los siguientes 4 pasos de una investigación ${ }^{12}$ : a) proceso de investigación, incluyendo diseño del estudio y concepción de la idea central, planteamiento y diseño de la investigación, análisis e interpretación de los datos o recopilación de datos u otro material; b) Trabajo sustancial en escribir el manuscrito o revisando el reporte científico, con importante contribución intelectual en escribir el manuscrito o parte de él, o ya sea, al revisar el manuscrito y realizar importantes modificaciones en su contenido; c) Aprobación de la versión final del manuscrito; d) Ser responsable y ser capaz de dar cuenta de todos los aspectos relacionados con la veracidad e integridad de la publicación. El que realiza sólo alguna de esas funciones dentro de la investigación no debería ser considerado como autor del manuscrito, pero incluido en la lista de agradecimientos.

Desafortunadamente, a pesar de existir definiciones claras, se describe malas prácticas en relación a las autorías, como son los autores invitados, ya sean por amistad o por coerción, y los autores fantasma. Se define como autores invitados y honorarios la inclusión como autor a un profesional sin que haya tenido participación en la ejecución ni redacción del trabajo publicado. Esta nominación puede obedecer a temas de conveniencia mutua o amistad, cuando los miembros de un equipo hacen acuerdos para aumentar las publicaciones respectivas. Una autoría por coerción ocurre cuando, por ejemplo, el jefe de un Laboratorio o Departamento exige ser incluido en las publicaciones, sin haber tenido participación directa. Por otro lado, el problema de autores fantasmas se ha descrito en grandes compañías, en que se elimina de la lista de autores a alguno de los investigadores para ocultar el conflicto de intereses ${ }^{13}$.

Basándose en encuestas a los investigadores, se han reportado prevalencias de 20 a $30 \%$ de autores honorarios y/o fantasmas en manuscritos publicados en revistas médicas de alto factor de impacto ${ }^{14}$, siendo más frecuente esta práctica en la publicación de casos clínicos o series de casos. Según Al-Herz y cols., las principales razones para incluir autores honorarios son el compromiso $(39,4 \%)$, para evitar conflictos $(16,1 \%)$, y aumentar la probabilidad de aceptación del manuscrito $(7,2 \%)^{15}$.

Para evitar los conflictos de autoría, habituales dentro del grupo de investigación ${ }^{16}$, se recomiendo acordar el orden de los autores previo a la redacción del manuscrito. En revistas biomédicas generalmente el orden de los autores obedece la lógica de colaboración relativa, teniendo en cuenta que muchas veces el último autor, o el autor de correspondencia son los que supervisan el trabajo del grupo. Cuando los conflictos aparecen después de haber enviado el manuscrito a la revista, los editores se basan en las guías del COPE para su resolución ${ }^{10}$.

\section{Conflicto de intereses}

Se consideran conflictos de intereses en la publicación cuando uno de los autores tiene algún tipo de relación personal o laboral que pudiera influencias su postura frente al problema planteado. Los conflictos de intereses se pueden dar también a nivel de revisores y editores, siendo fundamental declararlo ${ }^{11}$.

En una encuesta realizada a 3167 médicos de 6 especialidades, Campell y cols. reportaron que el $94 \%$ de los profesionales manifiestan algún tipo de relación con la industria farmacéutica, como es el recibir comida en su lugar de trabajo (83\%), aceptar muestras médicas (78\%), obtener financiamiento para congresos de la especialidad o educación continua (35\%) o pago por asesorías, conferencias, o enrolamiento de pacientes en ensayos clínicos $(28 \%)^{17}$. Si bien no está claro como estas relaciones influyen en las conductas médicas y divulgación científica, representa un desafío para el profesionalismo medico tanto a nivel de investigación como en el cuidado y protección de los intereses de los pacientes ${ }^{18}$. Se ha demostrado una asociación significativa entre el financiamiento de la industria y las conclusiones pro-industria del manuscrito ${ }^{19,20}$.

Sin duda, una adecuada declaración de conflicto de intereses, no elimina el problema, pero permite a los lectores balancear los resultados y formarse su propia opinión ${ }^{11}$.

\section{Duplicación, redundancia y plagio}

La palabra "plagio" deriva de las raíces latinas "plagium" que significa secuestrar o hurtar, por lo tanto, corresponde a la apropiación de ideas o palabras de otros autores, haciendolo pasar como propias. La Comisión de Ética de la Facultad de Medicina de la Universidad de Chile define como plagio "la apropiación, presentación y utilización de material intelectual ajeno, sin el debido reconocimiento de su fuente original. Constituye, por lo tanto, un acto fraudulento, en el cual existe presunción de intencionalidad, en el sentido de hacer aparecer un determinado conocimiento, labor o trabajo, como producto propio; y de desconocer la participación de otros en su generación, aplicación o en su perfeccionamiento" ${ }^{21}$. Se considera plagio la duplicación en forma parcial o total de una publicación previa, ya sea de otros autores o del mismo autor (auto-plagio).

El plagio es una amenaza común a la publicación cientifica, más aún en la era del "copy-paste”. Sin embargo su detección es un desafío constante para los 
editores. En un estudio de Higgins y colaboradores, en que evaluaron el plagio en manuscritos publicados en revistas biomédicas de especialidad, encontraron que el $17 \%$ de los manuscritos publicados tenían niveles de copia $^{22}$.

Actualmente hay varios programas disponibles para la detección de plagio. Recientemente Revista Chilena de Pediatría se suscribió al programa Crosref, que señala la posible duplicación del material contenido en un manuscrito, indicando tanto los párrafos copiados, como la fuente original. No obstante, cuando el plagio es realizado traduciendo manuscritos desde otros idiomas, su detección es más compleja, siendo pesquisados generalmente por revisores muy prolijos y familiarizados en el tema. Dado que aún no está definido cuál es el número aceptable de palabras copiadas, es fundamental el criterio del editor en el análisis.

El plagio muchas veces no es realizado en forma intencional, sino que por desconocimiento por parte de los autores. Un manuscrito puede ser reproducido parcial o completamente si cuenta con la autorización explícita de los editores, en ese caso se habla de publicación secundaria, práctica que se ha utilizado para tener acceso a audiencias de diferentes idiomas. No obstante, cuando no se cuenta con dicha autorización, la publicación duplicada significa un delito por cuanto viola la ley de derechos de autor y distorciona los resultados de meta-analisis posteriores ${ }^{23}$.

Lamentablemene, muchas veces el plagio es detectado después de la publicación del manuscrito. En ese caso los editores deben retractar la publicación del manuscrito junto con la recomendación de no citar a la publicación plagiada ${ }^{24}$.

\section{Comentarios finales}

Se estima que menos del 15\% de las investigaciones que se ejecutan llegan a publicarse ${ }^{25}$. Así por ejemplo, de los resúmenes presentados en reuniones científicas internacionales, aproximadamente la mitad son publi$\operatorname{cados}^{16}$, tasa que desciende aún más al considerar los resúmenes que se presentan en jornadas Latinoamericanas $^{26,27}$. Las causas de ello son la falta de motivación, escases de tiempo, los conflictos de autoría y problemas con el patrocinador ${ }^{16,25}$.

Por otro lado, las tasas de rechazo de los manuscritos recibidos en revistas de mayor prestigio científico alcanzan cifras tan importantes como 80-90\%. Un porcentaje importante de estos rechazos ocurre cuando el manuscrito es recibido por los editores, siendo las principales causas para ello la calidad editorial del manuscrito, su adecuación a la temática de la revista y, la detección de problemas éticos como la falta de respaldo por parte del comité de ética institucional, posible plagio y otras malas prácticas que pueden ser detec- tadas ya sea por el editor o por los pares evaluadores ${ }^{28}$. Describiéndose además una serie de sesgos editoriales editoriales en relación a la posibilidad de aceptación de los manuscritos ${ }^{29}$.

Para disminuir la probabilidad de rechazo, se recomienda a los autores ser honestos e íntegros en el proceso de investigación, revisar que el manuscrito sea adecuado a la revista que se está enviando y que cumpla las indicaciones editoriales, las que están detalladas en la guía para los autores ${ }^{30}$.

$\mathrm{Al}$ someter un manuscrito a una revista con comité editorial, se solicita a los autores firmar una carta de compromiso que incluye la declaración de la fuente de financiamiento de la investigación y potenciales conflictos de interés, la colaboración de cada uno de los autores en el desarrollo del manuscrito y el compromiso respecto a la originalida del mansucrito, para lo cual se debe asegurar que este no ha sido publicado previamente y que no ha sido enviado a otras revistas para considerar su publicación. Además se solicita declarar el respaldo del Comité de ética y consentimiento/asentimiento informado (ambos documentos se deben adjuntar). Los editores de las revistas biomédicas deben asegurar que el manuscrito publicado cumpla con los requisitos básicos de calidad, veracidad e integridad, sin llegar a ser jueces sobre el tema.

Ante la sospecha de una posible transgresión ética de un manuscrito ya publicado, los editores comienzan una investigación basada en las recomendaciones del COPE para deliberar acerca de la resolución del conflicto. En la institución patrocinadora del estudio es donde se debe realizar la investigación y eventual sanción de los autores que han realizado malas prácticas éticas en el proceso de investigación y publicación del manuscrito ${ }^{11}$.

La ética, así como las buenas y malas prácticas son inherentes a la conducta humana, y atraviesan a los investigadores tanto en su rol de autores, así como también a los revisores y editores, grupo al que ha definido Valderrama J. como "la trinidad necesaria para la buena divulgación del conocimiento"31. Estamos convencidos que las malas prácticas ocurren principalmente por desconocimiento más que por intensión de fraude ${ }^{32}$.

Revista Chilena de Pediatría no ha sido exenta a este tipo de conflictos. Por este motivo, esperamos que el presente manuscrito logre instruir y sensibilizar a nuestros investigadores, respecto a las bunas prácticas en la investigación y publicación, y, contribuir, en lo posible, a prevenir que estas acciones ocurran en los manuscritos enviados a nuestra Revista.

\section{Conflicto de intereses}

La autora declara no tener conflicto de intereses. 


\section{Referencias}

1. Elizondo-Rivera RL, Bosques-Padilla FJ. Cómo escribir un artículo científico: cómo lograr que su investigación sea publicada. Rev Gastroenterol Mex. 2007;72(2):113-6.

2. Villagran A, Harris P. Algunas claves para escribir correctamente un artículo científico. Rev. Chil. Pediatr. 2009;80(1):70-8.

3. Martinson BC, Anderson MS, de Vries R. Scientists behaving badly. Nature 2005;435(7043):737-8.

4. Fanelli D. How Many Scientists Fabricate and Falsify Research? A Systematic Review and Meta-Analysis of Survey Data. PLoS ONE 2009;4(5):e5738.

5. Matías-Guiu, J, García-Ramos R. Fraude y conductas inapropiadas en las publicaciones científicas.Neurología. 2010;25(1):1-4

6. Donoso E. Publica o perece: paradigma del fraude científico. Rev Chil Obstet Ginecol 2007;72(4):203-4.

7. Wager E, Williams P. Why and how do journals retract articles? An analysis of Medline retractions 1988-2008. J Med Ethics. 2011;37(9):567-70.

8. Steen RG, Casadevall A, Fang FC. Why Has the Number of Scientific Retractions Increased?. PLOS ONE 2013; 8(7): e68397.

9. Stretton S, Bramich N, Keys J, et al. Publication misconduct and plagiarism retractions: a systematic, retrospective study. Curr Med Res Opin. 2012;28(10):1575-83.

10. Committee of Publication Ethics (COPE) for request of addition of extra author after publication [Accedido el 21 de diciembre de 2018] disponible en https:// publicationethics.org/files/Full\%20set $\% 20$ of\%20English\%20flowcharts_9Nov2016. pdf

11. Reyes H. Problemas éticos en las publicaciones científicas. Rev Med Chile 2018;146:373-8.
12. Committee of Medical Journal Editors. Defining the Role of Authors and Contributors. [Accedido el 21 de diciembre de 2018] disponible en http:// www.icmje.org/recommendations/ browse/roles-and-responsibilities/ defining-the-role-of-authors-andcontributors.html

13. Von Oetinger A,Sadarangani K, Salas S. Conflictos éticos en las autorías de trabajos científicos. Rev Med Chile 2016; 144(11):1473-8.

14. Wislar JS, Flanagin A, Fontanarosa PB, DeAngelis CD. Honorary and ghost authorship in high impact biomedical journals: a cross sectional survey. BMJ 2011;343:d6128.

15. Al-Herz W, Haider H, Al-Bahhar M, Sadeq A. Honorary authorship in biomedical journals: How common is it and why does it exist? J Med Ethics. 2014;40(5):346-8.

16. Sprague S, Bhandari M, Devereaux PJ, et al. Barriers to full-text publication following presentation of abstracts at annual orthopaedic meetings. JBJS 2003;85(1):158-63

17. Campbell EG, Gruen RL, Mountford J, Miller LG, Cleary PD, Blumenthal D. A national survey of physicianindustry relationships. N Engl J Med 2007;356(17):1742-50

18. Brennan TA, Rothman DJ, Blank L, et al. Health industry practices that create conflicts of interest: a policy proposal for academic medical centers. JAMA 2006;295(4):429-33.

19. Bekelman JE, Li Y, Gross CP. Scope and impact of financial conflicts of interest in biomedical research: a systematic review. JAMA 2003;289(4):454-65.

20. Bhandari M, Busse JW, Jackowski D, Montori VM. Association between industry funding and statistically et al significant pro-industry findings in medical and surgical randomized trials. CMAJ 2004;170(4):477-80.

21. Comisión de Ética de la Facultad de
Medicina de la Universidad de Chile. En referencia al plagio intelectual (Documento). Rev Med Chile 2008;136:653-8.

22. Higgins JR, Lin F-C, Evans JP. Plagiarism in submitted manuscripts: incidence, characteristics and optimization of screening-case study in a major specialty medical journal. Res Integr Peer Rev. 2016;1(1):13

23. Habibzadeh F, Winker MA. Duplicate publication and plagiarism: causes and cures. Notfall Rettungsmed 2009;12:4158.

24. Reyes $\mathrm{H}$. El plagio en publicaciones científicas. Rev Med Chile 2009; 137:7-9.

25. Song F, Loke Y, Hooper L. Why Are Medical and Health-Related Studies Not Being Published? A Systematic Review of Reasons Given by Investigators. PLoS ONE 2014;9(10): e110418.

26 Elizondo C, Giunta D, González B, De Quirós F, Dawidowski A, Figar S, et al. La investigación clínica en residencias de medicina interna de la Argentina. Facilitadores y barreras. Medicina (Buenos Aires) 2012;72(6):455-60.

27. Fernandes FAMH, Ventura DE, Del Grande JC. Índice de publicação dos trabalhos apresentados no XXIV Congresso Brasileiro de Cirurgia. Rev Col Bras Cir. 2003;30(5):392-5.

28. Sá J. Rejeição e Indexação. Medicina Interna. 2018;25(1):5-6.

29. Matias-Guiu J, García-Ramos R. Sesgos en la edición de las publicaciones científicas. Neurología 2011;26(1):1-5.

30. Schonhaut L, Millán T, Podestá L. Revisión por pares: evidencias y desafíos. Rev Chil Pediatr 2017;88(5):577-81.

31. Valderrama J. Aspectos éticos en las publicaciones de revistas científicas de corriente principal. Rev Chil Pediatr. 2012;83(5): 417-9.

32. Brice J, Bligh J, Bordage G, et al. Publishing ethics in medical education journals. Academic Medicine 2009;84(10):S132-4. 